Revue internationale P.M.E.

Économie et gestion de la petite et moyenne entreprise

Revue

internationale

PME

\title{
Profils stratégiques des firmes de services aux entreprises
}

\section{Pierre-Yves Léo}

Volume 6, numéro 3-4, 1994

URI : https://id.erudit.org/iderudit/1008232ar

DOI : https://doi.org/10.7202/1008232ar

Aller au sommaire du numéro

Éditeur(s)

Presses de l’Université du Québec

ISSN

0776-5436 (imprimé)

1918-9699 (numérique)

Découvrir la revue

Citer cet article

Léo, P.-Y. (1994). Profils stratégiques des firmes de services aux entreprises. Revue internationale P.M.E., 6(3-4), 59-89. https://doi.org/10.7202/1008232ar

\section{Résumé de l'article}

Cet article présente les résultats d'une analyse de l'ensemble des firmes de services aux entreprises françaises de plus de vingt salariés. L'originalité de l'approche vient de ce que chaque entreprise est analysée à l'aide de ses données comptables rassemblées par l'Enquête annuelle d'entreprisesservices de l'INSEE. La méthodologie fait largement appel à l'analyse de données et, notamment, à l'analyse typologique. Les profils types relevés exploitent le fait que certaines attitudes stratégiques des firmes se traduisent, en fin de compte, par des résultats économiques et par des types d'organisation que l'on peut caractériser statistiquement.

Deux types de résultats apparaissent dans l'article: l'analyse des profils apporte une connaissance plus riche et complémentaire à l'analyse traditionnelle par branche d'activité. En second lieu, les catégories repérées expriment bien l'existence de différenciations entre les firmes et, notamment, de caractères spécifiques au secteur des services aux entreprises.
Ce document est protégé par la loi sur le droit d'auteur. L'utilisation des services d’Érudit (y compris la reproduction) est assujettie à sa politique d'utilisation que vous pouvez consulter en ligne.

https://apropos.erudit.org/fr/usagers/politique-dutilisation/ 


\title{
Profils stratégiques des firmes de services aux entreprises
}

\author{
Pierre-Yves LÉO \\ Jean PHILIPPE* \\ Centre d'économie régionale \\ Université Aix-Marseille III
}

\begin{abstract}
RÉSUMÉ
Cet article présente les résultats d'une analyse de l'ensemble des firmes de services aux entreprises françaises de plus de vingt salariés. L'originalité de l'approche vient de ce que chaque entreprise est analysée à l'aide de ses données comptables rassemblées par l'Enquête annuelle d'entreprisesservices de l'INSEE. La méthodologie fait largement appel à l'analyse de données et, notamment, à l'analyse typologique. Les profils types relevés exploitent le fait que certaines attitudes stratégiques des firmes se traduisent, en fin de compte, par des résultats économiques et par des types d'organisation que l'on peut caractériser statistiquement.

Deux types de résultats apparaissent dans l'article: l'analyse des profils apporte une connaissance plus riche et complémentaire à l'analyse traditionnelle par branche d'activité. En second lieu, les catégories repérées expriment bien l'existence de différenciations entre les firmes et, notamment, de caractères spécifiques au secteur des services aux entreprises.
\end{abstract}

\begin{abstract}
This article provides an analysis of French producer services based on the annual statistical survey of INSEE. All firms of more than 20 employees are

* Les auteurs sont ingénieurs de recherches, enseignants à la Faculté d'économie appliquée de l'Université d'Aix-marseille III. Docteurs en économie régionale et urbaine, ils sont auteurs de nombreux articles et ouvrages sur le développement industriel, les services, l'internationalisation des économies, les métropoles et les PME. Pierre-Yves Léo s'est spécialisé dans le développement international et les stratégies de PME ; Jean Philippe, diplômé postuniversitaire de UCLA, enseigne en outre à l'IAE d'Aix-en-Provence. Il s'intéresse tout particulièrement à l'économie et au management des services ainsi qu'à leur rôle dans le développement des métropoles. Adresse: Centre d'Économie Régionale, 15-19, Allée Claude-Forbin, Espace Forbin, 13627 Aix-en-Provence, Cedex 1, France.
\end{abstract}


taken into account: the data is fairly representative of the French case. The method is a multidimensional cluster analysis in order to type the strategic profile of each firm. Two kinds of results are obtained:

- Profile analysis leads toward a better understanding of the sector than the traditional branch analysis.

- Strategic profiles focus on the specific features of producer service firms and show the existing differences between firms.

\section{RESUMEN}

Este articulo presenta los resultados de un anàlisis del conjunto de las firmas de servicios para las empresas francesas de màs de veinte asalariados. La originalidad de este enfoque radica en el hecho de que cada empresa se analisa gracias a datos contables reunidos por la Encuesta Anual sobre Empresas-Servicios del INSEE. La methodologia recurre ampliamente al anàlisis de datos y particularmento al anàlisis tipològico. Los perfiles tipos identificados explotan el hecho de que algunas actitudes estratégicas de las firmas se traducen por resultados economicos y por tipos de organizaciòn que se pueden caracterizar por la estadistica.

Aparecen dos tipos de resultados en el articulo: primero, el anàlisis de los perfiles permite un conocimiento màs rico et complementario al anàlisis tradicional por sector de actividad. Después, las categorias identificadas expresan muy bien la existencia de diferenciaciones entre las firmas y especialmente la de rasgos especificos del sector de los servicios para las empresas.

\section{Introduction}

Les dirigeants des entreprises de services sont conscients de la particularité de leur activité de prestataires et de la nécessité d'en tenir compte pour établir leur avantage comparatif sur le marché. Les recherches en stratégie s'attachent à montrer les particularités des entreprises de services (Thomas, 1978). La nature même du service et son mode de réalisation peuvent être vus comme des moyens pour une mobilité stratégique des firmes (Shostack, 1987). Mais l'importance du personnel en contact pour la réussite des prestations, la gestion des clients au cours du processus de servuction ${ }^{1}$, le choix et l'utilisation des technologies,

1. «Servuction»: ce néologisme a été forgé pour traduire la spécificité de la production des services : produit, produire, production a donné par analogie, service, servir, «servuction». Une définition couramment retenue est la suivante : "Action de mise en relation d'un prestataire avec un bénéficiaire pour obtenir un effet et dans laquelle le bénéficiaire joue un rôle actif.» (Eiglier et Langeard 1988) 
constituent également des facteurs de différenciation stratégique entre les entreprises (Heskett, 1990).

La recherche servant d'appui à cet article avait pour principal objectif de mettre en évidence des profils stratégiques types, statistiquement repérables ${ }^{2}$ par des informations accessibles sur les entreprises de services. Le champ de l'analyse était l'ensemble des firmes de plus de vingt salariés du secteur des services aux entreprises en France, soit environ 4000 firmes, dont $80 \%$ avaient moins de 200 salariés.

Les formulations théoriques font souvent l'hypothèse implicite que toutes les entreprises ont des capacités identiques pour mettre en œuvre leurs stratégies. Or, l'environnement de chaque entreprise est spécifique, mais surtout leur organisation ne leur confere pas une capacité identique pour affronter la concurrence. La recherche des profils n'est pas fondée sur l'agrégation des données d'entreprise par secteur ou par taille, mais sur des ratios ${ }^{3}$ établis pour chaque entreprise. Le logiciel FASTCLUS de SAS, que nous avons utilisé, permet d'opérer des regroupements de firmes dont les ratios sont les plus proches. Compte tenu du nombre des observations, la classification s'opère par grappes. Les profils types constituent un instrument utile de compréhension et de connaissance des forces et des mécanismes qui animent le secteur des services aux entreprises, car ils permettent de mieux tenir compte de la diversité des comportements des firmes.

Cet article présente d'abord notre modèle d'analyse et les critères retenus, en référence aux approches déjà développées sur les stratégies des services. La seconde partie analyse les caractéristiques des sept archétypes relevés de firmes. La dernière partie se propose d'évaluer l'influence qu'exerce le secteur sur les profils stratégiques repérés.

2. C'est-à-dire au moyen des informations disponibles dans l'Enquête annuelle d'entreprises (EAE) - Services.

3. L'Enquête annuelle d'entreprises - Services de l'INSEE contient une foule de données qualitatives et quantitatives sur chaque entreprise. Cet outil d'information de premier ordre a pu être utilisé ici grâce à la collaboration de la Direction des activités tertiaires de l'INSEE qui gère cette enquête de bout en bout. Les données individuelles des firmes sont protégées par le secret statistique, ce qui impose aux chercheurs d'obtenir tout d'abord une habilitation pour y accéder, puis d'en respecter les clauses, c'est-à-dire, principalement, de ne pas publier ni divulguer d'informations concernant un trop petit nombre d'entreprises. 


\section{Modèle d'analyse, indicateurs et critères}

James Heskett (1990) souligne, à juste titre, que la définition des stratégies dans le secteur des services est un exercice difficile. Les premières recherches dans ce domaine ont voulu mettre en valeur les différences entre les stratégies des firmes industrielles et celles des entreprises de services. Les principales dimensions stratégiques spécifiques aux services ont ainsi été inventoriées tandis que, simultanément, des efforts de classification sectorielle devaient être menés ${ }^{4}$ du fait de l'indigence des nomenclatures existantes. L'aspect conceptuel apparaît, dans toutes ces recherches, étroitement imbriqué avec les méthodologies qui permettent la mise en évidence des stratégies.

\subsection{Références de l'analyse stratégique}

L'élaboration de stratégies ne peut être dissociée de l'analyse de la capacité des firmes à les mettre en œuvre. Mais les fondements de cette capacité ainsi que les critères de son évaluation font l'objet de divergence entre les auteurs. Lentz (1980), dans un essai de synthèse, propose de retenir trois dimensions pour déterminer cette capacité : le savoir-faire technique de la firme, la capacité à acquérir et à créer des ressources économiques et la gestion de la technologie. D'autres points de vue opèrent fréquemment la distinction entre les entreprises qui assoient leur avantage comparatif sur le personnel ou le facteur humain, en général, et celles qui misent plutôt sur la technologie ou l'équipement. Ainsi, une synthèse de James Heskett propose l'existence de trois points de focalisation

4. Notre travail n'a pas échappé à cette règle : une première étape a été l'élaboration d'une nomenclature sectorielle adaptée aux services aux entreprises à partir des informations détaillées fournies par l'EAE-Services sur l'activité des firmes. Quatre grands secteurs y ont été définis : ils regroupaient les 34 branches élémentaires utilisées (le détail de celles-ci peut-être consulté sous le graphique $\mathrm{n}^{\circ} 2$ ). La logique du regroupement en quatre secteurs découlait de la nature du service, de la forme qu'il prend et du contenu de la prestation tels qu'ils ont été analysés par Barcet, Bonamy et Mayère (1983). Trois catégories étaient proposées : services de conseils et études (exemples : ingénierie, consultance), services de mise à disposition de moyens (exemples : locations de machines, de main-d'œuvre), services de « faire » (exemples : gardiennage, comptabilité). Nous avons divisé en deux cette dernière catégorie pour tenir compte du contenu plutôt matériel ou intellectuel de la prestation :

- le «faire matériel» ou «faire d'exécution», regroupant nettoiement, gardiennage et sécurité, routage, secrétariat, travaux à façon, etc.

- le «faire intellectuel» ou «faire d'assistance», regroupant comptabilité, facturation, renseignements commerciaux et enquêtes, formation, coordination ou pilotage de chantier, etc. 
des stratégies de services: le positionnement par rapport à la concurrence, la recherche d'effets de levier, la recherche d'efficacité dans la servuction. Cette analyse, très concrète et très proche des préoccupations des dirigeants d'entreprises, permet de bien situer l'analyse typologique que nous avons menée.

- Le positionnement de la firme concerne des stratégies purement commerciales lui permettant de se différencier de ses concurrents. Les travaux de Shostack (1987), Eiglier et Langeard (1988), entre autres, se rattachent à ce courant de recherches. Les nombreuses stratégies de ce type qui ont été inventoriées ne peuvent cependant pas être repérées au moyen d'une analyse statistique des comptes des entreprises et se dérobent donc à toute tentative de repérage systématique.

- Les stratégies de recherche d'effet de levier, tout comme celles d'intégration du système de servuction au système de gestion, touchent par contre à la structure et au fonctionnement des firmes. Leur objectif est d'identifier les améliorations ou les innovations nécessaires au développement de la firme. Les travaux sur la qualité (Parasuraman et al., 1988 ; Zeithmal et al., 1990; Grönross, 1988), sur la gestion des ressources humaines (Schneider, 1973), sur l'optimisation des rencontres de services (Czepiel et al., 1985; Bitner, 1990) et ceux sur l'impact de la technologie (Quinn, 1988) concernent ce type de stratégies. On peut y ajouter la plupart des travaux sur la localisation des firmes (Coffey et Polese, 1984) et sur les réseaux de services (Léo et Philippe, 1991a). De telles stratégies ont des conséquences comptables repérables. Le personnel et la technologie figurent dans les comptes des firmes, par la masse salariale, les catégories de salariés, le montant et la nature des immobilisations, des investissements, etc.

\subsection{Critères et indicateurs}

Le choix des critères de classification est essentiel dans toute démarche typologique. Ici, les critères d'analyse devaient permettre de situer l'entreprise dans le contexte stratégique de son développement. Quatre ont été retenus:

- l'ouverture internationale, indice de compétitivité et de capacité organisationnelle de la firme;

- la compétence et le niveau du personnel, indice de choix organisationnels, de spécification du concept autour d'un type de prestation et d'image;

- la rentabilité, indice de performance, d'efficacité et de capacité à dégager du profit; 
- le dynamisme technologique, indice d'une stratégie de développement à base d'équipements neufs incorporant les dernières technologies disponibles sur le marché.

Ces quatre critères ont été évalués à l'aide des indicateurs suivants :

- Le taux d'exportation (d'après le chiffre d'affaires réalisé avec l'étranger) a été considéré, malgré ses défauts, comme une indication intéressante de l'ouverture internationale des firmes. Faute d'autres informations, les autres formes de l'internationalisation des services, telles que l'établissement à l'étranger ou les accords contractuels de type franchise, n'ont pu être pris en compte. En outre, pour certaines activités de service, il est impossible d'exporter et l'indicateur prend donc, a priori, des valeurs nulles dans ces cas-là. Sur le plan statistique, ce critère est encore plus sélectif que pour le secteur manufacturier, car il concerne uniquement un aspect très avancé des stratégies de développement extensif à base commerciale.

- Le taux de cadres (proportion de la main-d'œuvre, salariée ou non) fournit un bon indicateur pour le second critère. Il permet d'évaluer correctement le degré d'engagement des firmes dans les stratégies axées sur la compétence ou le savoir-faire de haut niveau. Mais il reste muet en ce qui concerne leur orientation précise ou leur contenu.

- Le taux de résultat a été utilisé dans sa version « résultat net d'exploitation rapporté à la valeur ajoutée» pour mesurer les performances des firmes en termes de rentabilité et de profit. Les différents taux de rendement du capital calculés ne se sont pas montrés directement utilisables, du fait de leur propension à produire des valeurs extrêmes, liées au très faible engagement capitalistique d'un bon nombre de firmes de service. Par ailleurs, l'évaluation nette du résultat d'exploitation est préférable à l'évaluation brute qui, en incluant le poids des investissements passés (amortissements), introduit une relation avec l'intensité capitalistique de la servuction.

- L'indicateur de dynamisme technologique a été construit en rapportant les investissements en machines et outillages neufs au capital de chaque entreprise. La dimension du capital a été étendue aux actifs d'exploitation (stocks) de manière à compenser certaines tendances aux valeurs extrêmes. Cet usage se justifie, car la grandeur ainsi calculée constitue la partie la moins liquide de l'actif, celle qui est gelée pour réaliser le cycle d'exploitation et que l'acquisition de nouveaux équipements vient augmenter. Pour réduire le caractère fluctuant de l'investissement, qui n'était disponible que sur une seule année, cet indicateur comporte également au numérateur l'amortissement, témoin des investissements passés de toute nature. 
Les quatre indicateurs ainsi définis se montrent statistiquement indépendants : chacun fournit une information différente des autres, les coefficients de corrélation, deux à deux, étant tous très voisins de zéro. L'élaboration d'une classification des firmes à partir d'eux s'avère donc statistiquement pertinente.

Une batterie de critères très similaires avait déjà été utilisée sur le champ des PME françaises du secteur manufacturier (Léo, 1986 et 1987). Neufs profils typiques y avaient été relevés, manifestant le positionnement des firmes sur un axe stratégique dominant (parmi les trois ou quatre possibles) avec des degrés d'engagement variables. Des situations stratégiques et des choix effectués en matière de gestion ou de développement ont ainsi pu être décrits et l'indépendance de ces profils par rapport à la branche précise d'activité a pu être observée.

\section{Sept catégories de situations stratégiques}

Sans entrer dans le détail de la méthodologie employée 5 , il convient de préciser que de nombreux essais de classification ${ }^{6}$ ont permis de déterminer que les meilleures partitions s'obtiennent avec sept catégories de firmes.

Plusieurs essais ont été tentés avec six classes seulement, dans l'espoir de réduire le nombre de classes à gérer ensuite. Aucune de ces partitions ne s'est montrée supérieure à ce qui peut être obtenu avec sept classes. Une des classifications à six types s'élève cependant bien au-dessus des autres du même rang: cette typologie parvient à diviser en deux la classe la plus nombreuse, qui regroupe les deux tiers ou les trois quarts des firmes. Cela révèle l'importance de cette subdivision pour la qualité de la partition. Avec sept classes, cette grosse classe centrale est toujours éclatée en deux, ce qui explique, en partie, l'avantage systématique de ces classifications sur celles à six types.

Parmi toutes les classifications opérées pour obtenir sept catégories, l'une affichait des performances un peu supérieures aux autres et a donc été retenue. L'incertitude liée à la méthode de partition est cependant assez faible: sur l'ensemble des classifications en sept types, $95 \%$ des entreprises restent affectées à la même classe, l'amélioration obtenue sur la meilleure partition

5. Celle-ci fait l'objet d'une présentation étendue dans le rapport de recherche de Léo et Philippe (1991b).

6. Le nombre élevé des essais de classification est rendu nécessaire par la méthode de regroupement des firmes qui aboutit à des variantes selon les noyaux de départ et l'ordre dans lequel les observations sont lues. Cette incertitude est inhérente à la dimension du fichier étudié. 
provenant du reclassement de $5 \%$ des firmes. Ces sept catégories ont été baptisées en fonction de leurs caractères dominants. Il est intéressant d'analyser d'abord la façon dont cette partition découpe le champ étudié, pour vérifier, ensuite, à travers la description détaillée des classes d'entreprises, que celles-ci correspondent bien à des profils types de situations stratégiques.

\section{GRAPHIQUE 1}

Valeurs prises par les indicateurs ${ }^{7}$ mesurant la qualité de chaque partition opérée, selon le nombre de classes demandées

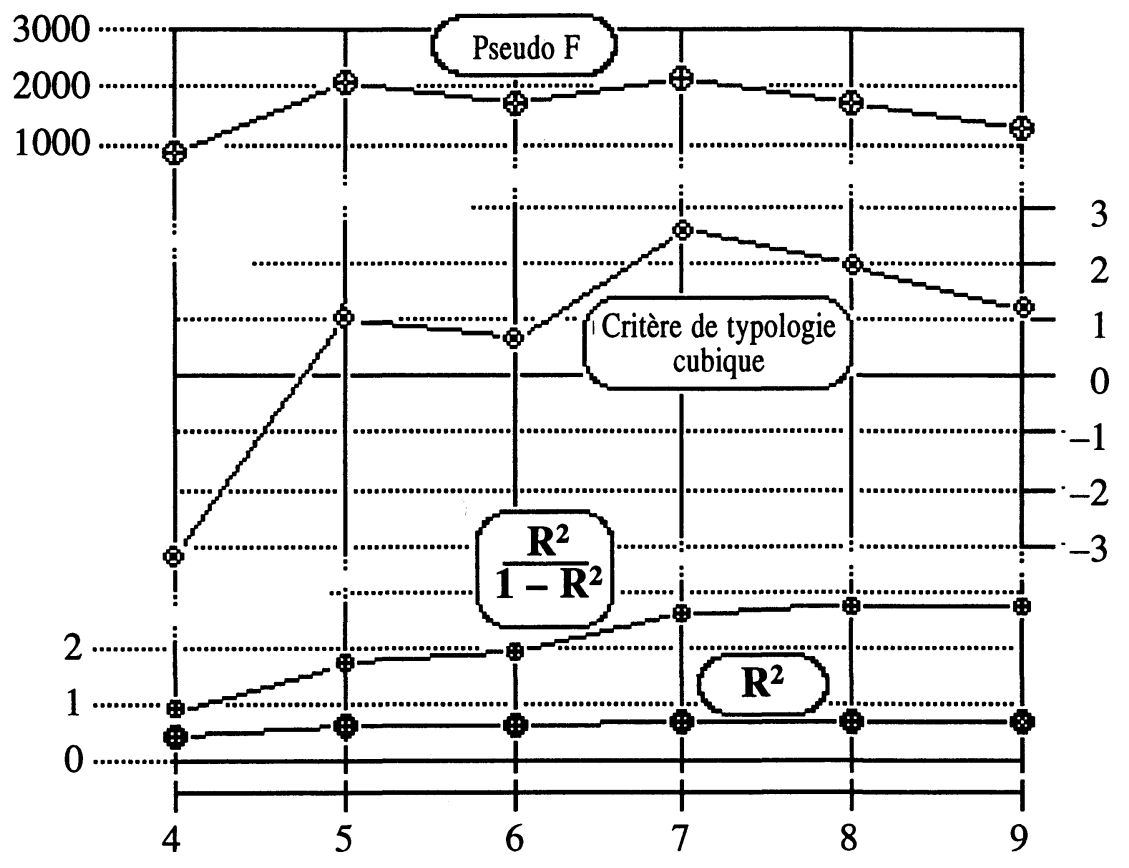

Nombre de classes établies sur 4915 firmes

7. Ces quatre indicateurs sont proposés par le logiciel FASTCLUS de SAS :

- Le $\mathrm{R}^{2}$ indique le pouvoir explicatif de la partition si l'on estime chaque observation (firme) par la valeur moyenne des ratios de sa classe de rattachement.

- Le ratio $R^{2} /\left(1-R^{2}\right)$ est proposé comme amplificateur de la sensibilité du $R^{2}$, mais il n'apporte aucune information supplémentaire.

- Le pseudo $\mathrm{F}$ introduit la dimension « $\mathrm{n}$ » du fichier traité et le nombre de classes demandées « $\mathrm{c} »$ : $\quad \mathrm{R}^{2}(\mathrm{n}-\mathrm{c}) /(\mathrm{c}-1)\left(1-\mathrm{R}^{2}\right)$

- Le «critère de typologie cubique» (cubic clustering criterion) est plus complexe; il n'est significatif que si les quatre critères sont indépendants statistiquement (ce qui est ici le cas). Plus sa valeur est élevée, plus la partition est statistiquement satisfaisante. Pour plus de détails, voir Milligan et Cooper (1983). 


\subsection{Caractéristiques de la partition et sélectivité des critères}

Les nombreux essais de typologie ont également permis de mieux cerner la forme du nuage formé par les entreprises dans «l'hyper-espace» à quatre dimensions, défini par la combinaison des quatre axes indépendants correspondant chacun à un indicateur de la classification : une forte densité au centre, se diluant progressivement lorsqu'on s'en éloigne, pour se focaliser autour de quelques groupements proches des axes ou situés entre eux. Le poids du centre avait déjà pu être observé pour le secteur manufacturier. Mais l'emplacement du centrage ainsi que l'importance des groupes combinant plusieurs axes stratégiques apparaissent comme des caractères originaux du secteur tertiaire étudié.

\section{TABLEAU 1}

Valeurs moyennes des quatre indicateurs pour les sept classes

(Valeurs calculées sur les entreprises n'ayant subi aucune évaluation, redressement ou limitation sur les données utilisées par les ratios de tri.)

\begin{tabular}{cccccc}
\hline $\begin{array}{c}\text { Nombre } \\
\text { total } \\
\text { d'entreprises }\end{array}$ & $\begin{array}{c}\text { Désignation } \\
\text { du } \\
\text { type }\end{array}$ & $\begin{array}{c}\text { Taux } \\
\text { d'exportation } \\
(\mathbf{\%})\end{array}$ & $\begin{array}{c}\text { Pourcentage } \\
\text { de } \\
\text { cadres }\end{array}$ & $\begin{array}{c}\text { Indicateur } \\
\text { de dynamisme } \\
\text { technologique }\end{array}$ & $\begin{array}{c}\text { Ratio } \\
\text { de résultat } \\
(\%)\end{array}$ \\
\hline 344 & Compétence & $(3,0)$ & $\mathbf{8 5 , 4}$ & 0,15 & $(+12,6)$ \\
81 & Compétence & $(5,3)$ & $\mathbf{6 6 , 6}$ & $\mathbf{0 , 8 1}$ & $(+13,9)$ \\
& -techno & & & & \\
895 & Composite & $(1,0)$ & 37,9 & 0,12 & $\mathbf{+ 1 5 , 3}$ \\
92 & Export & $\mathbf{7 5 , 1}$ & 47,5 & $(0,12)$ & $(+0,05)$ \\
220 & Techno & $(0,4)$ & 11,9 & $\mathbf{0 , 7 4}$ & $\mathbf{( + 9 , 5 )}$ \\
2217 & Moyen & $(0,4)$ & 8,5 & 0,13 & $+10,0$ \\
121 & Pertes & $(4,6)$ & 39,0 & 0,10 & $\mathbf{- 3 8 , 0}$ \\
3970 & Champ total & 2,7 & 25,0 & 0,70 & $+\mathbf{7 , 7}$ \\
\hline
\end{tabular}

(Les chiffres en gras soulignent les caractéristiques des classes, ceux entre parenthèses indiquent les valeurs peu représentatives: lorsque l'écart type de la classe est supérieur ou égal à la moyenne.)

Le tableau 1 indique les valeurs moyennes des sept catégories de firmes. On trouve donc des classes très peu nombreuses, comptant moins d'une centaine de firmes, face à une classe centrale très dense regroupant plus de 2000 entreprises. Les dominantes stratégiques de ces groupes sont fréquemment concentrées autour d'un seul indicateur, mais deux classes sont nettement multi-axiales: les types «composite» et «compétence-techno». L'identification des types de firmes s'est faite à partir des moyennes de chaque indicateur pour chaque classe. Elle a été confirmée par la façon dont la typologie répartit les firmes selon les tranches de valeur d'un seul indicateur: c'est ce que montrent les quatre 
diagrammes suivants qui permettent de lire l'existence de valeurs-seuil à partir desquelles on ne trouve plus aucune firme d'une certaine classe. Ces diagrammes montrent aussi le pouvoir séparateur des différents indicateurs et révèlent ainsi la hiérarchie opérationnelle des critères.

1) Le taux d'exportation semble être le critère le plus sélectif. Il tranche nettement le champ vers la valeur de $45 \%$ : au-dessus, on ne trouve quasiment que les firmes classées très exportatrices, au-dessous, on n'en trouve plus aucune.

\section{Diagramme 1}

\section{Répartition des firmes selon les classes et selon les valeurs prises par le taux d'exportation}
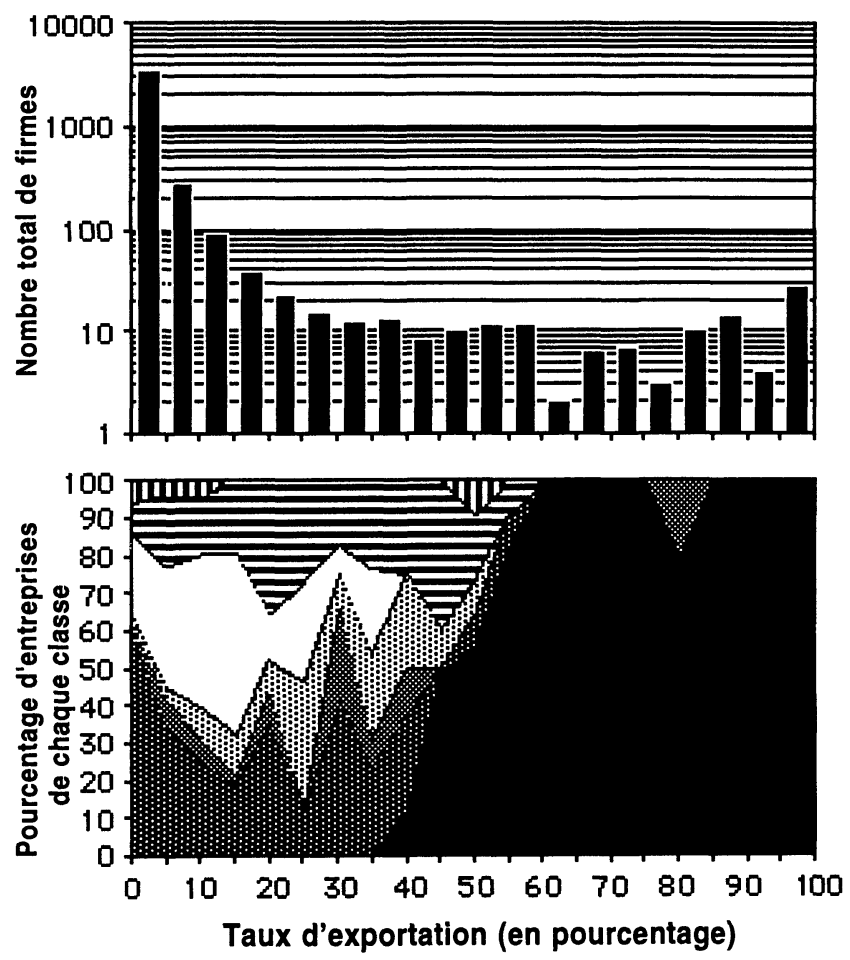

\begin{tabular}{|l|}
\hline \multicolumn{1}{|c|}{ LÉGENDE } \\
Type MOYEN \\
$\square$ Type COMPOSITE \\
曰 Type COMPÉTENCE \\
Iㅣ Type TECHNO \\
Type PERTES \\
$\square$ Type EXPORT \\
Type COMPÉTENCE \\
et TECHNO
\end{tabular}

Indépendamment des réserves que cet indicateur appelle pour les services, il faut remarquer que la séparation ne s'est pas faite entre les firmes exportatrices et les autres, qui sont la grande majorité, mais entre les firmes les plus engagées à l'export et les autres, y compris celles qui ont déjà un certain chiffre d'affai- 
res avec l'étranger. Le second point est que le seuil de $45 \%$ est sensiblement plus élevé que le seuil de 30/35\% qui prévalait pour les PME industrielles.

2) Le taux d'encadrement de la main-d'œuvre apparaît comme le second grand critère de séparation des firmes. Il divise le champ en trois groupes distincts. Au-dessous de 25/30 \%, les firmes où domine l'apport de main-d'œuvre, avec ou sans investissement technologique. De 25/30 à 60/70\% des structures intermédiaires, composites, associent un encadrement développé à un personnel subalterne nombreux, parfois avec un comportement d'équipement en surcroît. Au-dessus de 60/70\% de cadres, ce sont des firmes qui ont clairement opté pour le savoir-faire de haut niveau et la compétence, en l'associant ou non à un renforcement de l'équipement. Seul ce type avait pu être repéré pour les PME manufacturières, l'indicateur utilisé alors étant plus indirect (niveau de salaire moyen).

Diagramme 2

Répartition des firmes selon les classes et selon les valeurs prises par le taux d'encadrement de la main-d'œuvre
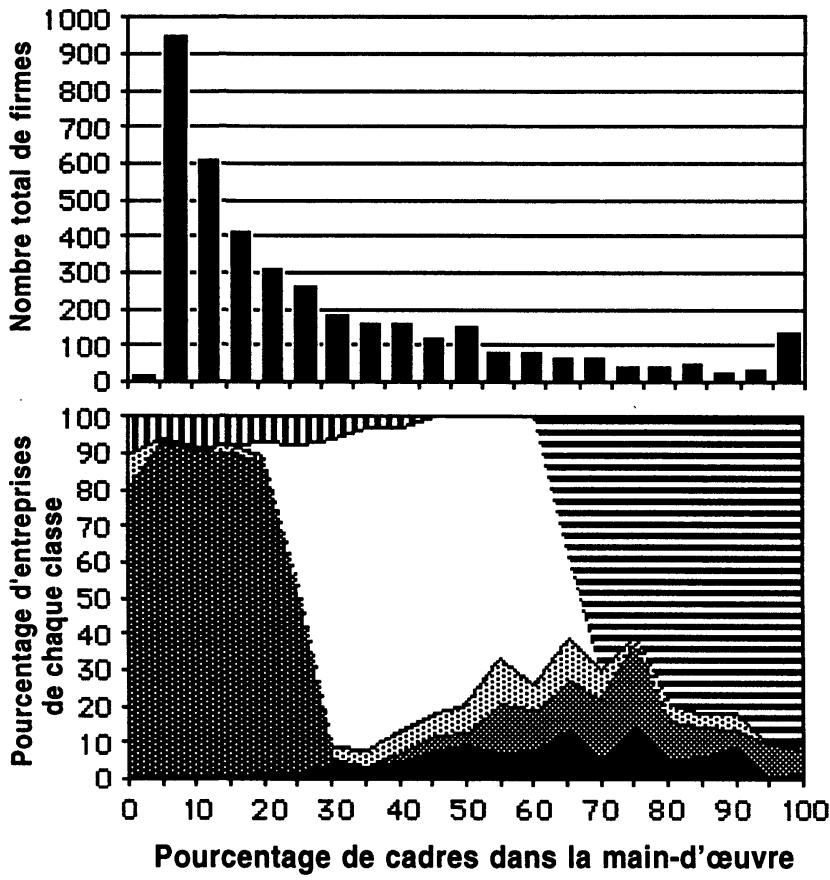

\begin{tabular}{|c|}
\hline \multicolumn{1}{|c|}{ LÉGENDE } \\
Type MOYEN \\
$\square$ Type COMPOSITE \\
日 Type COMPÉTENCE \\
III Type TECHNO \\
哴 Type PERTES \\
$\square$ Type EXPORT \\
Type COMPÉTENCE \\
et TECHNO \\
\hline
\end{tabular}


3) L'indicateur de dynamisme technologique contribue aussi à structurer la typologie, mais de façon moins nette, comme le montre l'importance des lignes horizontales dans le diagramme. Aux alentours de $50 \%$, il sépare les firmes ayant un comportement d'investissement en machines des autres. Elles appartiennent à deux catégories: les plus nombreuses n'ayant pas d'autre engagement stratégique visible; le second groupe, associant un fort taux d'encadrement à sa dynamique d'équipementation.

DiAGRAMme 3

Répartition des firmes selon les classes et selon les valeurs prises par l'indicateur de dynamisme technologique
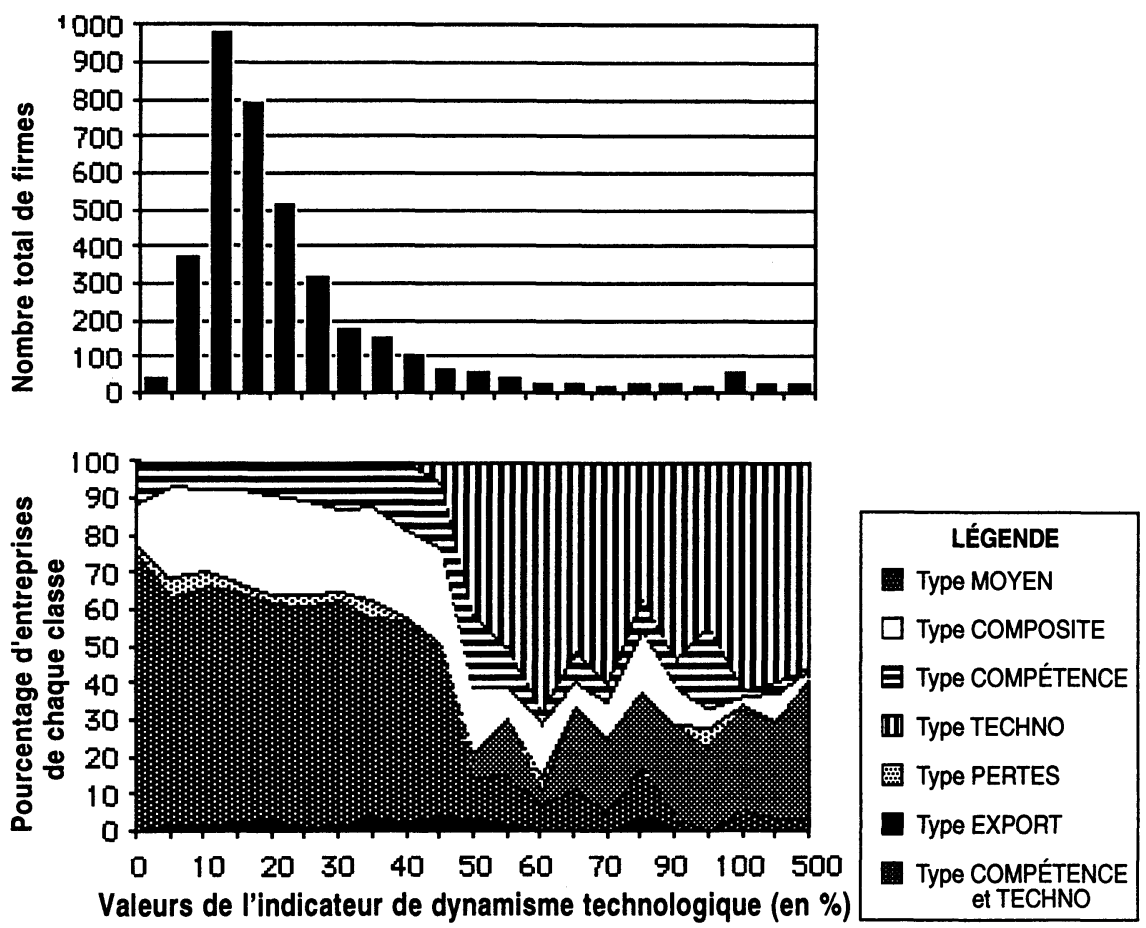

4) Les résultats obtenus par l'entreprise ne constituent pas un critère puissant de tri, sauf en ce qui concerne les valeurs les plus négatives. Cette conclusion avait déjà été vérifiée dans le seul secteur manufacturier, bien que, dans ce contexte, ait également émergé une classe typée seulement par ses excellents résultats. Ici, c'est la classe composite qui se distingue par de meilleurs rendements, mais sa caractérisation par le 
taux d'encadrement paraît plus nette. Les performances en termes de résultats semblent être la conséquence d'une forme efficace d'organisation du travail et de l'intégration de niveaux différenciés de compétence, au moins autant que d'une stratégie de rentabilisation et de profit qui conduirait à limiter le recours aux emplois les plus coûteux pour utiliser le plus possible du personnel subalterne. Il faut aussi noter que le profil central s'étend, pour les services, jusqu'aux rentabilités les plus fortes. Dans le secteur manufacturier, cette classe centrale était nettement cantonnée aux rentabilités moyennes ou faibles. Les services aux entreprises paraissent donc peu influencés, dans leur positionnement stratégique, par un résultat qui est, dans la grande majorité des cas, confortablement positif (une firme sur dix, seulement, enregistre une perte nette, tandis qu'une sur trois produit un excédent net dépassant $10 \%$ de la valeur ajoutée).

\section{Diagramme 4}

Répartition des firmes selon les classes et selon les valeurs prises par le taux de résultats
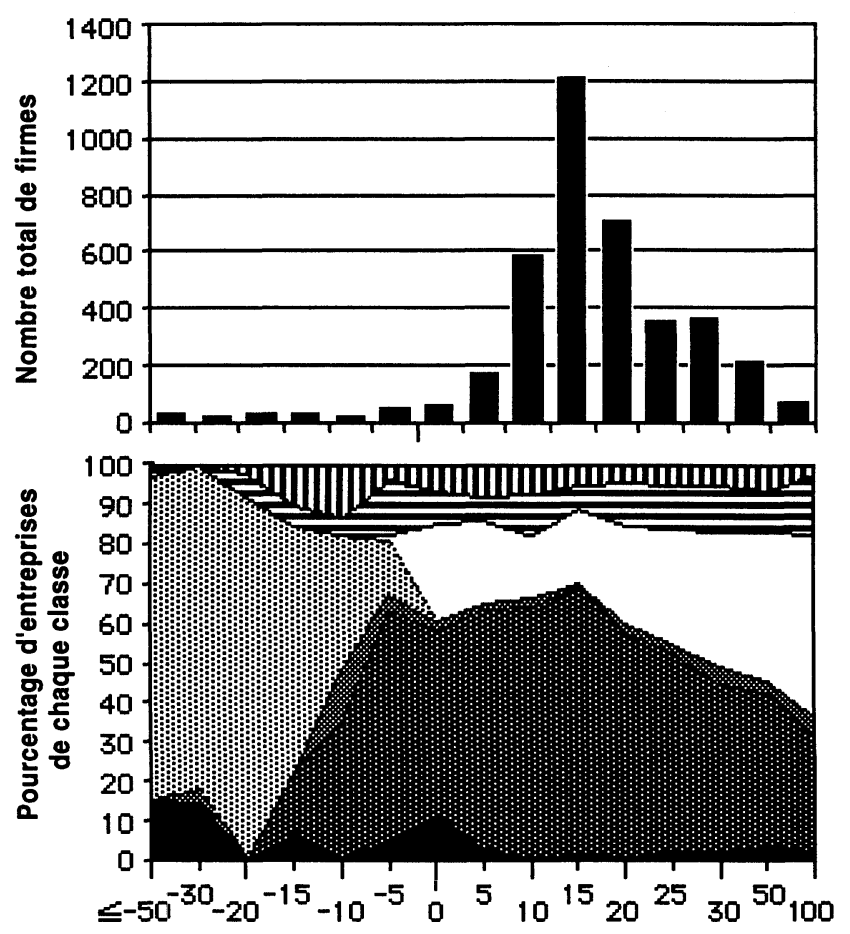

Ratio d'excédent net d'exploitation / Valeur ajoutée (en \%)

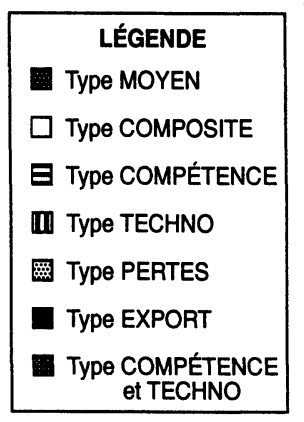


Une typologie simplifiée, construite sur une hiérarchie des critères, peut être comparée à celle obtenue directement de façon multicritère. La forme la plus approchante ressemble assez à la typologie pour mériter l'intérêt: 300 firmes seulement s'y trouvent déplacées d'une classe à une autre. Cette classification «manuelle» distingue en premier lieu et successivement les classes EXPORT et PERTES, selon le seul critère qui les définit vraiment: des ventes à l'étranger comptant pour plus de $45 \%$ de leur chiffre d'affaires pour les premières, des pertes nettes supérieures à $20 \%$ de leur valeur ajoutée pour les secondes. C'est ensuite une imbrication des deux derniers critères qu'il a fallu construire pour rester fidèle à la typologie de référence, avec, pour chaque indicateur, des seuils de séparation différents selon les plages de variation de l'autre. C'est dire combien la typologie associe étroitement ces différents critères sur le champ tertiaire étudié, de façon bien plus claire et plus évidente qu'elle ne le faisait pour les PME du secteur secondaire.

\subsection{Les sept profils types}

Les profils mis à jour ne méritent cette appellation que dans la mesure où ils décrivent des types de situation stratégique. Cela doit se traduire par un ensemble de spécificités assez riche et complexe. À cet égard, il est significatif que, selon le profil type auquel elles sont rattachées, les firmes adoptent des comportements préférentiels dans de nombreux autres domaines. Certes, ces particularités ne sont pas toujours très tranchées et ne concernent jamais la totalité des indicateurs disponibles ${ }^{8}$, mais l'assortiment même des indicateurs significativement différents de la moyenne, propre à chaque classe, personnalise déjà le profil type. L'étude des spécificités des différentes classes permet ainsi de dresser un portrait archétypal des sept situations stratégiques repérées. L'ordre dans lequel elles se présentent ci-après est arbitraire, mais il débute par les profils les plus significatifs d'une stratégie de ressources humaines, firmes du type «compétence» et «compétence-techno», puis celles du type «composite». À cette logique peuvent aussi se rattacher, indirectement, les types «export». Viennent ensuite les types «techno» et «moyen» qui ont certaines ressemblances entre eux. Les profils «pertes » sont présentés en dernier, il s'agit moins d'un profil caractéristique que de firmes dont les comptes sont rendus difficilement interprétables du fait de très fortes pertes enregistrées sur l'exercice étudié.

\subsubsection{Les firmes du type “COMPÉTENCE»}

Les 344 firmes classées dans cette catégorie sont caractérisées par un seul critère dominant: elles emploient une forte proportion de main-d'œuvre de type cadre,

8. La liste des ratios calculés est fournie en annexe. 
mettant ainsi l'accent sur un développement à base de compétences de très haut niveau. Les cadres représentent, pour ce profil, $85 \%$ de l'effectif total en moyenne (60\% au minimum et jusqu'à $100 \%)$, chiffre qu'il convient de rapprocher des $25 \%$ observables sur la moyenne du champ étudié et des 10 à 15\% fréquemment observés dans le secteur industriel. Plutôt de petites dimensions, ces firmes rassemblent $30 \%$ du potentiel de cadres des services aux entreprises. Une part significative de ceux-ci ne sont pas salariés, mais relèvent de sociétés professionnelles où plusieurs confrères indépendants (professions libérales) s'associent. Les firmes du type «compétence» réalisent des chiffres d'affaires et créent des valeurs ajoutées conséquentes pour leur taille, mais restent peu développées en termes de capital. On y rencontre spécifiquement des firmes géographiquement compactes ou ayant simplement créé un second établissement.

Elles obtiennent des résultats positifs supérieurs à la moyenne $(+12,8 \%$ contre $+7,7 \%$ ) sans doute dus à une productivité élevée, supérieure de $50 \%$ à la moyenne du secteur ( $300 \mathrm{kF}$ contre $193 \mathrm{kF}$ par personne). Les rémunérations versées sont, naturellement, élevées. L'appel aux achats intermédiaires (36\% du CA) reste important pour le secteur tertiaire, limitant la valorisation ${ }^{9} \mathrm{du}$ chiffre d'affaires. Cela peut être rapproché de l'importance relative des stocks de produits vendus à l'appui du service.

Ce profil est principalement constitué par des services de conseils et études qui représentent $51 \%$ de ces firmes. Toutes les activités de ce secteur ne sont pourtant pas concernées. Les plus spécifiques sont l'ingénierie informatique (76 firmes, soit $22 \%$ du profil), les conseils en organisation et gestion ( 28 entreprises), l'architecture ( 15 firmes), ainsi qu'une activité classée avec le secteur de mise à disposition de moyens: la location de matériels informatiques et de moyens de calculs (14 entreprises). À l'évidence, la compétence du personnel est ici une condition d'accès au marché qui l'emporte sur le profil des métiers de la location. On trouve également, mais de façon non spécifique, de nombreuses entreprises de ce profil dans des branches des secteurs de «faire», comme en comptabilité (31) et en travaux à façon informatiques (26).

\subsubsection{Les firmes du type "COMPÉTENCE-TECHNO»}

Les firmes se rattachant à ce profil sont peu nombreuses (81). Il apparaît comme une variante du profil «compétence» dont il se distingue principalement par un investissement important par rapport à un capital de départ généralement faible.

9. La valorisation du chiffre d'affaires est décrite par le ratio valeur ajoutée/chiffre d'affaires. 
Cet investissement concerne à $83 \%$ le matériel et l'outillage, traduisant une volonté d'équipement de ces entreprises. L'emploi des cadres y est un peu moins poussé (67\% en moyenne, mais s'échelonnant de 40 à $100 \%)$ et la taille des firmes, en termes d'emplois et en capital immobilisé, y est plus faible. Elles réalisent pourtant des chiffres comparables de ventes et de valeur ajoutée. Plus encore que les précédentes, elles sont concentrées sur un seul établissement, parfois deux.

Elles obtiennent de très bons résultats $(+14 \%)$ surtout si l'on tient compte que ce ratio est net d'amortissement et caractérise donc ce qui reste après le financement de leur effort d'accroissement du capital. Cela s'accompagne de hautes rémunérations liées à une productivité élevée ( $308 \mathrm{kF}$ par tête). Elles se comportent souvent en donneurs d'ordres vis-à-vis de confrères, pratiquant une sous-traitance qui concerne presque $9 \%$ de leur chiffre d'affaires. C'est un des facteurs expliquant la valorisation moyenne qu'elles réalisent à partir de leur chiffre d'affaires $(61 \%)$. Certaines sont engagées sur des marchés étrangers, mais de façon modérée ( $5 \%$ du CA en moyenne).

Ce sont à peu près les mêmes secteurs que précédemment qui sont concernés par ce profil, mais de manière plus concentrée : 52 des 81 firmes (64\%) sont des services d'études ou de conseil. Parmi celles-ci, la moitié (26 entreprises) relèvent de l'ingénierie informatique, 7 , du conseil en organisation et gestion, et 6 , de l'ingénierie du bâtiment.

\subsubsection{Les firmes du type “COMPOSITE»}

Cette classe est très caractéristique des services aux entreprises (et probablement des services en général). S'y rattachent 895 entreprises qui se distinguent ainsi du groupe central «moyen», lequel domine la typologie par le nombre. Le critère le plus apparent qui les caractérise est un résultat net d'exploitation très positif ( $+15,6 \%$ en moyenne). Mais, à l'analyse, ce critère paraît associé au taux de main-d'œuvre cadre dont les valeurs moyennes-hautes sont caractéristiques. Employant en moyenne $38 \%$ de cadres, les firmes de ce profil manifestent un engagement modéré sur une stratégie de compétence. Tous leurs taux sont compris entre $25 \%$ et $65 \%$ de cadres (diagramme 2).

Il semble que ces entreprises réalisent des compromis (heureux, si l'on en juge par les résultats) sur plusieurs axes stratégiques: des rémunérations élevées, rentabilisées par une bonne productivité ( $268 \mathrm{kF}$ par tête), un engagement capitalistique et des investissements notables. Les firmes de ce profil conservent une taille moyenne ou petite; elles ont développé, plus que les autres, des réseaux de taille modeste ( 2 à 5 établissements), mais captent des chiffres d'affaires déjà appréciables sans recourir à l'exportation. Elles sont amenées à rétrocéder un certain nombre de leurs marchés ou à sous-traiter une partie du 
travail à des confrères. Cela concerne presque $7 \%$ de leur chiffre d'affaires. Leur capacité de valorisation reste moyenne, ce qui traduit la nécessité d'engager des frais significatifs pour assurer le service.

Elles se trouvent réparties dans un peu tous les types de secteurs, avec une prédominance significative pour les services à contenu intellectuel (conseils, études, «faire d'assistance») qui regroupent près de $70 \%$ des stratégies «composites ». Mais les affinités sectorielles de ce profil sont très diverses, puisqu'on y retrouve 24 activités sur les 34 que compte la nomenclature. Toutes les activités rattachées aux services à contenu intellectuel sont présentes, à l'exception de la facturation et de la gestion de commandes. Les branches les plus marquées par ce type de stratégie sont les conseils juridiques et fiscaux (où ce profil représente $75 \%$ des firmes), la création, le conseil et le courtage en publicité $(69 \%)$, la production d'articles publicitaires (52\%), l'ingénierie du bâtiment et des travaux publics $(48 \%)$, les «autres ingénieries » $(44 \%)$, la régie publicitaire et la location d'espaces publicitaires ( $43 \%)$, etc. L'énumération montre, une fois encore, que, pour les situations stratégiques des firmes, les segments de marché (l'informatique, la publicité) ont un pouvoir structurant qui peut l'emporter sur la nature des services proposés (conseils, études, location, assistance, exécution).

\subsubsection{Les firmes du type "EXPORT»}

Exporter est une des façons d'élargir son marché. Pour les firmes de services aux entreprises, ce n'est certainement pas la plus facile, sauf pour des cas très particuliers. À la difficulté de vendre à distance des prestations immatérielles s'ajoutent, en effet, les difficultés liées à la communication internationale (Philippe, 1990) alors que, précisément, la qualité de transmission des informations (émission, réception, compréhension correcte et complète des messages) est cruciale pour le marketing des services. Les firmes, peu nombreuses, qui relèvent ce défi réalisent de bonnes performances à l'exportation (plus de $45 \%$ de leur chiffre d'affaires). Cela manifeste l'existence d'un axe de développement de la firme qui va bien au-delà de la simple saisie d'opportunités ou d'occasions lorsqu'elles se présentent. Il s'agit de comportements affirmés et concentrés dans ce champ: $78 \%$ des exportations de services aux entreprises sont accomplies par ces 92 firmes qui ne représentent que $30 \%$ des exportateurs (et $2 \%$ de toutes les entreprises) du secteur tertiaire étudié. La question centrale pour ce type de stratégie est de bien évaluer sur quoi porte l'avantage compétitif qui permet l'accès à des marchés lointains et, dans un second temps, si les conditions de la prestation permettent une exploitation rentable.

L'engagement à l'exportation s'accompagne le plus souvent d'une taille assez élevée, quelle que soit la dimension retenue (effectif employé, chiffre d'affaires, valeur ajoutée ou capital immobilisé). C'est donc une stratégie 
difficile pour les PME de services, plus encore que pour les PME industrielles. Cette stratégie paraît exclusive d'une croissance par épuisement du marché national, car peu de ces firmes ont plus d'un ou deux établissements sur le territoire français. Elles présentent, par contre, une organisation typique qui les différencie des autres firmes observées:

- un fort potentiel de cadres (47\% des effectifs), très bien rémunérés (mieux, en moyenne que ceux des profils précédents) et rarement non salariés;

- un recours systématique aux formes d'emploi à court terme (location, contrats à durée déterminée). Ce type d'emploi représente pour l'ensemble des services aux entreprises $7 \%$ de l'effectif total, mais il atteint $14 \%$ pour ces firmes exportatrices, allant jusqu'à constituer 25 à $50 \%$ de la main-d'œuvre dans certains cas. Cela indique sans doute que, malgré son importance, la pénétration de ces firmes sur les marchés étrangers conserve un caractère aléatoire et fluctuant;

- une valorisation faible pour les services (53\%) qui les rapproche de la moyenne des PME manufacturières (46\%). L'importance des achats est à rapprocher de deux autres caractéristiques : l'importance des stocks et de la sous-traitance d'honoraires ( $12 \%$ du CA en moyenne). Le service, pour s'exporter, s'associe à des biens matériels vendus avec lui;

- un capital important et des investissements fonciers significatifs qui montrent clairement l'association d'une politique d'image à la politique d'exportation;

- une productivité élevée ( $299 \mathrm{kF}$ par tête) qui permet à la plupart des entreprises de ce profil d'aboutir à des résultats positifs. Mais quelquesunes affichent des pertes catastrophiques qui les rapprochent de la catégorie «pertes».

La concentration des stratégies d'exportation sur certains secteurs est évidente : $79 \%$ (73 sur 92 ) relèvent du secteur des conseils et études. Plus précisément encore, ce sont quatre secteurs d'ingénierie (BTP, industrie, autres et contrôles-expertise) qui sont seuls concernés. Cela tient à plusieurs facteurs :

- certains services ne peuvent être exportés pour des raisons de cadre juridique ;

- d'autres affronteraient des différences culturelles brouillant leur capacité de communication (ce qui est moins marqué dans le domaine technique);

- d'autres ne présentent pas un avantage compétitif marqué, ou en tout cas perceptible, vis-à-vis de la concurrence à l'étranger. Cela concerne la plupart des services de «faire matériel»; 
- pour d'autres, enfin, l'exportation représenterait un risque majeur, sans mesure avec les bénéfices pouvant en être attendus (mise à disposition de moyens matériels).

\subsubsection{Les firmes du type "TECHNO »}

Les 220 firmes de cette classe s'engagent dans une stratégie équipementière, acquérant des machines ou outillages neufs dans des proportions élevées eu égard à leur capital de départ. Ce type d'investissement («machines») représente $87 \%$ de leur intensification capitalistique (contre $67 \%$ pour la moyenne des services aux entreprises). Ces chiffres sont à rapprocher de ceux observables chez les firmes industrielles qui ne réalisent aussi que $65 \%$ de leurs investissements sous forme de machines et outillages. Partant d'une capitalisation faible, les entreprises du profil «techno» effectuent des investissements qui vont souvent au-delà du doublement de leurs immobilisations.

Elles ne ressemblent que peu aux firmes classées «compétence-techno» qui adoptent une stratégie voisine dans le domaine de l'investissement. À l'inverse, elles se différencient peu, à l'exception des investissements, du groupe suivant qui rassemble les firmes de profil «moyen». Elles s'en distinguent par une taille plutôt moyenne ou faible, par la rareté des entreprises à réseau d'établissement et par des résultats un peu inférieurs, ce qui est à nuancer par le fait que ces résultats sont mesurés nets des amortissements qui sont élevés pour cette classe. La valorisation y est forte, $74 \%$ du chiffre d'affaires étant de la valeur ajoutée produite par l'entreprise. La productivité du travail y est basse $(149 \mathrm{kF}$ par personne), ce qui traduit des activités de main-d'œuvre avec un encadrement faible, de l'ordre de $10 \%$; cela permet aussi d'interpréter leur appel à l'équipement comme une «industrialisation » du service qui tend à être assuré par un couple homme-machine. Autre caractère original, ces firmes emploient une forte proportion de main-d'œuvre à temps partiel ( $25 \%$ en moyenne), ce qui confère une certaine souplesse à leurs possibilités d'offre.

Ce profil se retrouve surtout dans les services de «faire matériel» $(52 \%$ des cas) et de mise à disposition (21\%). Il est particulièrement propre aux activités de nettoyage de locaux professionnels, de location de main-d'œuvre, de travaux à façon informatiques, de réalisation d'opérations publicitaires, de gardiennage, de transport de fonds et sécurité, de régie publicitaire. En outre, on trouve aussi des firmes de ce profil de façon typique dans les études économiques, sociologiques et de marché et, de manière non spécifique, en comptabilité. Sans doute s'agit-il d'entreprises ayant fait leur spécialité de la réalisation d'enquêtes pour les premières, de la saisie comptable pour les secondes. 


\subsubsection{Les firmes du type "MOYEN"}

C'est la classe la plus nombreuse, comptant 2217 entreprises, soit $56 \%$ du champ. Malgré ce nombre, les comportements de ces firmes sont très homogènes, décrivant bien ceux d'un centre compact du «nuage» des firmes. Dans l'ensemble, les caractères relevés sont proches de la moyenne générale, le nombre joue en ce sens, mais sur certains points, ce profil «moyen» est bien spécifique: peu de cadres $(8 \%)$, peu d'investissements, mais des résultats satisfaisants $(+10 \%)$; les effectifs employés sont souvent nombreux, mais les chiffres d'affaires, valeurs ajoutées et capitaux immobilisés sont faibles, en proportion. Une forte valorisation ( $77 \%)$ montre des activités à fort contenu immatériel, des services réalisés essentiellement par un facteur travail peu qualifié - comme le confirme la faiblesse des stocks -, un bas niveau de la productivité (143 kF par tête en moyenne) et un recours étendu aux formes d'emploi précaires qui représentent, groupées, $35 \%$ de l'effectif total : $9 \%$ sont pris en location ou sous contrat à durée déterminée tandis que $26 \%$ le sont à temps partiel (ces chiffres s'élèvent respectivement à $7 \%$ et à $18 \%$, en moyenne, pour l'ensemble du champ). Bien que la forme la plus courante soit l'entreprise mono-établissement, c'est dans ce type «moyen» que l'on trouve spécifiquement les réseaux les plus étendus et les plus denses.

On retrouve des firmes se rattachant à ce profil «moyen» dans toutes les activités, mais avec une nette prédominance dans les secteurs de «faire matériel » et de mise à disposition. Il faut noter la quasi-absence de ce profil parmi les conseils en organisation-gestion ( 9 cas seulement sur 75 firmes) et la location de moyens informatiques (5 cas sur 50). Pour les activités de conseil et études, le profil «moyen » représente globalement $22 \%$ des firmes; pour celles $\mathrm{du}$ «faire intellectuel» $50 \%$; pour le «faire matériel» $75 \%$ (1090 cas); et pour la mise à disposition, $66 \%$ (557 cas). Les activités les plus caractéristiques sont le nettoyage de locaux professionnels $(91 \%)$, la location de main-d'œuvre $(89 \%)$, le gardiennage, la sécurité, le transport de fonds $(85 \%)$, le routage $(77 \%)$, la location d'entrepôts $(75 \%)$, le travail de bureau à façon $(72 \%)$, la location de matériel de travaux publics et de levage (71\%), la location de biens d'équipement divers ainsi que la facturation et la gestion de commandes (67\%).

\subsubsection{Les firmes du type "PERTES»}

Les 121 firmes qui se retrouvent dans cette classe ont pour caractéristique commune un déficit comptable désastreux : en moyenne, $87 \%$ de la valeur ajoutée passent en pertes, avant même le prélèvement des charges financières. Il est difficile de considérer cette classe comme un archétype, dans la mesure où les causes d'un tel état peuvent être très diverses. Elles sont, en outre, bien difficiles 
à identifier dans des grandeurs comptables exagérément déséquilibrées, notamment une valeur ajoutée ridiculement faible, ne s'élevant, en moyenne, qu'à $37 \%$ du chiffre des ventes. Un certain nombre de traits leur sont cependant spécifiques:

Ce sont surtout des firmes de grande dimension, en particulier, en ce qui concerne le capital immobilisé qui semble extrêmement lourd pour ces entreprises. Peu de PME peuvent survivre à de tels déséquilibres. Un nombre significatif de ces grosses firmes ont développé des réseaux d'établissements étendus. La productivité du travail y est basse, malgré un encadrement et des niveaux de rémunérations supérieurs à la moyenne. En dépit de leur faible valorisation (ou en est-ce la cause ?), elles pratiquent la sous-traitance pour une partie non négligeable de leur chiffre d'affaires.

Elles sont nettement spécifiques aux secteurs d'activités de conseils et d'études (où $39 \%$ se regroupent), ainsi que de mise à disposition avec $35 \%$ des cas. Il s'agit surtout de l'ingénierie industrielle, de la promotion et administration d'infrastructures, ainsi que de la régie et location d'espaces publicitaires.

\section{Profils stratégiques et secteurs d'activité}

Une hypothèse essentielle, à peu près vérifiée pour les PME manufacturières, est que la logique sectorielle n'explique pas seule les profils types: les situations stratégiques dépendent au moins autant des facteurs propres à chaque firme (de leur taille, de leur organisation et de leur localisation, notamment) que de leur secteur qui ne définit qu'une partie de leur environnement.

La description détaillée des profils types montre l'entretien de rapports avec l'activité de service exercée par l'entreprise. La question est d'évaluer correctement ces rapports : le secteur exerce-t-il un effet dominant sur les situations stratégiques identifiées ou bien les spécificités observées sont-elles le fruit de contextes plus ou moins favorables, selon les secteurs, à tel ou tel profil type ? Enfin, une logique plus macrosectorielle intervient-elle et retrouve-t-on les mêmes axes stratégiques dans le secteur industriel?

\subsection{De grandes familles de branches}

$\mathrm{Au}$ niveau des quatre grands types de secteurs, la répartition des profils types est assez explicite, mais les plus fortes spécificités concernent les profils les moins nombreux, ce qui, somme toute, est assez naturel. Le tableau 2 indique les caractéristiques de cette répartition. 
Tableau 2

Nombre de firmes de chaque profil type selon leur secteur

\begin{tabular}{lrrrrrrrrrr}
\hline $\begin{array}{l}\text { Secteur } \\
\text { Profil } \\
\text { type }\end{array}$ & $\begin{array}{c}\text { Activités } \\
\text { de conseils } \\
\text { et études }\end{array}$ & \multicolumn{2}{c}{$\begin{array}{c}\text { Activités } \\
\text { de faire } \\
\text { d'assistance }\end{array}$} & \multicolumn{2}{c}{$\begin{array}{c}\text { Activités } \\
\text { de faire } \\
\text { d'exécution }\end{array}$} & \multicolumn{2}{c}{$\begin{array}{c}\text { Activités } \\
\text { de mise }\end{array}$} & \multicolumn{2}{c}{ à disposition } & \multicolumn{2}{c}{ TOL } \\
\hline Compétence & $\mathbf{1 7 5}$ & $\mathbf{5 0 , 9}$ & 47 & 13,7 & 68 & 19,8 & 54 & 15,7 & 344 & 100,0 \\
Comp.-Techno & $\mathbf{5 2}$ & $\mathbf{6 4 , 2}$ & $\mathbf{7}$ & 8,6 & 10 & 12,4 & 12 & 14,8 & 81 & 100,0 \\
Composite & $\mathbf{3 5 5}$ & $\mathbf{3 9 , 7}$ & $\mathbf{2 6 6}$ & $\mathbf{2 9 , 7}$ & 148 & 16,5 & 126 & 14,1 & 895 & 100,0 \\
Export & $\mathbf{7 3}$ & $\mathbf{7 9 , 4}$ & 10 & 10,9 & $\mathbf{8}$ & $\mathbf{8 , 7}$ & 1 & 1,1 & 92 & 100,0 \\
Pertes & $\mathbf{4 7}$ & $\mathbf{3 8 , 8}$ & 11 & 9,1 & 21 & 17,4 & $\mathbf{4 2}$ & $\mathbf{3 4 , 7}$ & 121 & 100,0 \\
Moyen & 204 & 9,2 & 366 & 16,5 & $\mathbf{1 0 9 0}$ & $\mathbf{4 9 , 2}$ & $\mathbf{5 5 7}$ & $\mathbf{2 5 , 1}$ & 2217 & 100,0 \\
Techno & 30 & 13,6 & 29 & 13,2 & $\mathbf{1 1 4}$ & $\mathbf{5 1 , 8}$ & $\mathbf{4 7}$ & $\mathbf{2 1 , 4}$ & 220 & 100,0 \\
Ensemble & 936 & 23,6 & $\mathbf{7 3 6}$ & 18,5 & 1459 & 36,8 & 839 & 21,1 & 344 & 100,0 \\
\hline
\end{tabular}

(Les chiffres en gras indiquent les valeurs spécifiques.)

Les activités de conseil et d'étude sont bien différenciées des autres par le poids des cinq premiers profils. On pouvait prévoir ce résultat pour l'exportation et les stratégies de compétence. L'importance des stratégies «composites » est, par contre, intéressante: elle montre que, pour plus du tiers des firmes de conseils, la rentabilité passe par un développement de l'emploi non cadre. Les activités de «faire d'assistance» se déterminent typiquement pour des stratégies «composites» du même type, mais les profils bas des stratégies moyennes, sans être caractéristiques, y sont majoritaires. Ce profil «moyen » caractérise les deux derniers secteurs, en association avec le profil «techno». Malgré ces particularités, il n'y a pas d'exclusion systématique et l'on trouve, dans chaque secteur, des cas non spécifiques en nombres significatifs (sauf en ce qui concerne les deux profils les moins fréquents).

Si l'on pousse l'analyse à un niveau plus fin de la nomenclature, les grandes lignes de ce qui a été observé demeurent, à quelques exceptions près. On relève ainsi que le point commun des différents secteurs de conseils et études réside dans la spécificité de situations stratégiques de type «composite» et non de celles du type «compétence», «compétence-techno» ou «export» : les premières sont sous-représentées dans l'ingénierie industrielle, le contrôle technique et les conseils juridiques ou fiscaux; les secondes sont très spécifiques de l'ingénierie informatique, mais absentes ou presque du contrôle technique, des conseils juridiques ou fiscaux, des conseils ou créateurs en publicité, des autres ingénieries et de l'architecture; quant aux stratégies d'exportation, elles ne concernent que l'ingénierie, le contrôle technique et, dans une moindre mesure, les conseils en gestion et le marketing. 
Les activités rassemblées dans le secteur du «faire d'assistance», où la prestation est à dominante plus intellectuelle que matérielle, présentent un aspect contrasté. Une partie se rattache aux comportements typiques des conseils, avec la présence simultanée de stratégies tournées vers l'exportation, d'autres, du type «compétence» ou «composites» (assistance technique, pilotage de chantier, formation de personnel, renseignements commerciaux, enquêtes, recrutement). D'autres activités ressemblent fort aux secteurs de «faire d'exécution», avec des profils «moyens » prépondérants (facturation, gestion de commandes, assistance juridique et judiciaire). Deux secteurs, enfin, hésitent entre ces deux orientations : les métreurs-géomètres et la comptabilité où coexistent des stratégies à profil bas (type «moyen») et d'autres de type «composite».

Les services de «faire d'exécution » et ceux de mise à disposition, qui ont tous deux une dominante matérielle dans la prestation voient dominer, parfois de façon écrasante, le profil «moyen», associé parfois au profil «techno». On trouve ainsi le routage, tous les travaux à façon, les locations d'équipements (pour travaux publics ou divers), les entrepôts, où domine seul le profil «moyen». Il coexiste avec des profils «techno» dans le nettoiement, le gardiennage, la réalisation d'opérations publicitaires et la location de main-d'œuvre. Mais un certain nombre de branches, rattachées a priori à ces deux secteurs, voient leurs firmes développer des stratégies qui les apparentent à celles du secteur des conseils : les stratégies «composites » dominent dans la location de matériels ou de moyens informatiques; elles sont accompagnées d'un nombre spécifique de profils «compétence» pour les activités de production d'articles publicitaires, régie publicitaire et location d'espaces, promotion et administration d'infrastructures.

Les rapprochements entre la nomenclature et les profils types des firmes permettent de mieux comprendre le contenu des secteurs de services. Une typologie des secteurs peut être construite, à partir des comportements stratégiques des firmes de chaque secteur. Le résultat est résumé dans le graphique 2 où se croisent les spécificités observées des trois principaux profils types. Le positionnement relatif des secteurs permet d'établir trois grandes familles de secteurs, ainsi que quelques sous-familles.

La première grande division oppose les secteurs à profils types «moyens » aux autres. Ce premier groupe qui rassemble surtout des activités de prestation de moyens matériels (nettoyage, gardiennage, routage, facturation, travaux à façon, locations diverses) se lit dans la partie droite, et au fond, du graphique 2. Il voit se développer des satellites sur ses marges, dont les plus significatifs sont la comptabilité et les métreurs-géomètres.

La deuxième grande division isole les quatre secteurs où les stratégies de compétence sont très développées, ainsi que les stratégies «composites»: 
ingénierie informatique, conseils en organisation-gestion, architecture et location de matériels et de moyens informatiques. Ces quatre secteurs se trouvent à gauche et «en avant» dans le graphique 2 .

Le dernier groupe est surtout caractérisé par la faiblesse des profils «moyens » associée, la plupart du temps, à l'importance des profils «composites». Il regroupe la plupart des autres activités de conseil, ainsi qu'un certain nombre de satellites venant du «faire d'assistance» et de la publicité (production d'articles et régie).

Cette analyse suggère des reclassements sectoriels significatifs: des domaines ou des filières de services comme l'informatique où la publicité peuvent constituer des forces de cohérence équilibrant celles issues de la logique des segments de marchés ou des métiers concernés (ingénieurs, conseils, créateurs, loueurs, etc.) et dont il faudrait tenir compte au niveau des regroupements intermédiaires.

\subsection{Profils tertiaires et profils industriels}

Les profils types identifiés pour les services aux entreprises diffèrent sensiblement de ce qu'ils étaient dans le champ industriel: Alors que, pour ce dernier, les types décrits présentaient une apparence très unidimensionnelle, plusieurs des catégories émergeant du champ services sont, de manière explicite, multidimensionnelles.

Autre différence significative avec le secteur industriel: il est beaucoup plus délicat de retrouver la subdivision suggérée par Huppert (1981) entre des logiques stratégiques dominées par le souci du développement et d'autres dominées par celui de la rentabilité ou de la survie immédiate.

Le type «composite » qui associe de bons résultats à une bonne proportion de cadres dans l'effectif relève aussi bien d'un engagement stratégique modéré que d'une stratégie efficace de rentabilité. C'est cette classe dont l'émergence rend les typologies plus significatives, sur le champ des services. Elle sort du centre géant qui caractérise quasiment toutes les classifications comptant moins de sept classes. Cependant, sa frontière avec les types «moyens» est plus délicate à établir : $95 \%$ des cas d'instabilité recensés concernent cette classe «composite» et $72 \%$ la classe centrale résiduelle. Le gros des firmes de service de cette classe «moyenne» se rapproche plutôt des PME manufacturières «modérément bénéficiaires » que des «médiocres» qui formaient le centre du champ manufacturier et dont le principal problème est de survivre. Le tableau 3 propose une comparaison terme à terme des typologies obtenues sur les deux champs, à partir d'une méthode et d'indicateurs similaires. 


\section{GRAPHIQUE 2 \\ Spécificité des trois principaux profils selon les secteurs de services aux entreprises}

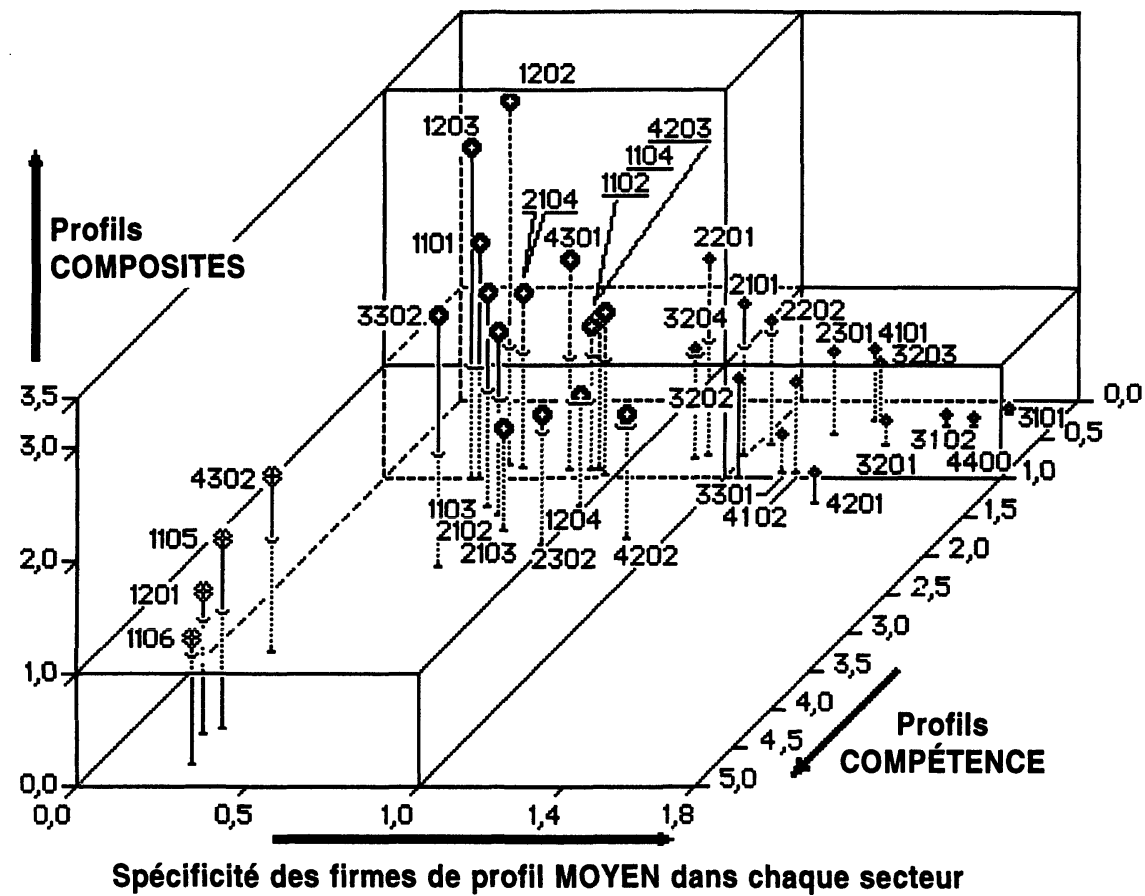

Rappel de la nomenclature

1101 Ingénierie du bâtiment et des travaux publics

1102 Ingénierie industrielle

1103 Autres ingénieries

1104 Contrôle technique, vérification, expertise

1105 Architecture

1106 Ingénierie informatique

1201 Conseils en organisation, gestion

1202 Conseils juridiques et fiscaux, expertise

1203 Création, conseils et courtage en publicité

1204 Études économiques, sociologiques, de marché

2101 Géometres

2102 Coordination, pilotage de chantier

2103 Assistance technique

2104 Formation de personnel

2201 Comptabilité

2202 Assistance juridique et judiciaire

2301 Facturation, gestion de commande

2302 Renseignements commerciaux, enquêtes, recrutement
3101 Nettoyage, entretien de locaux

3102 Gardiennage, surveillance, transp. de fonds

3201 Routage

3202 Travaux a façon informatiques

3203 Travaux à façon / bureaux (secrétariat, traduction, reprographie)

3204 Travaux à façon divers et mal identifiés

3301 Réalisation d'opérations publicitaires

3302 Production d'articles publicitaires

4101 Location de matériel TP et de levage

4102 Location de biens d'équipement divers

4201 Location d'entrepôts

4202 Location immobilière à usage professionnel

4203 Promotion, administration d'infrastructures

4301 Régie publicitaire, location d'espaces publicitaires

4302 Location de matériel informatique ou moyens de calcul

4400 Location de main-d'œuvre 
TABLEAU 3

Essai de regroupement des sept classes d'entreprises selon les dominantes stratégiques de type industriel

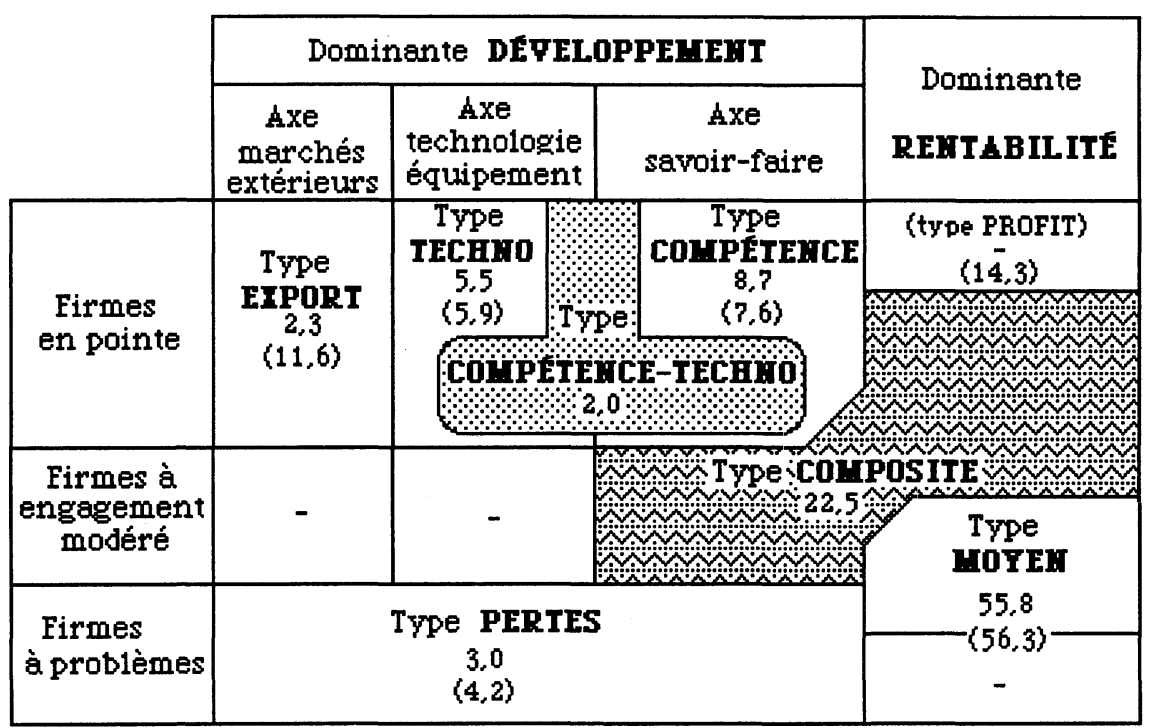

(Les chiffres indiquent les pourcentages de firmes concernées et, entre parenthèses, rappellent ce qu'ils étaient pour les firmes industrielles.)

La comparaison des fréquences des différents profils avec l'industrie est intéressante, pour autant que l'on puisse assimiler les profils les uns aux autres. Les firmes centrales seraient plus nombreuses dans les services ( $78 \%$ de type moyen ou composite, contre $56 \%$ de type central dans l'industrie), ainsi que les stratégies appuyées sur une compétence de haut niveau (11\% contre $8 \%)$ et, fait remarquable, celles à base de renforcement de l'équipement $(7,5 \%$ contre $5,9 \%)$. Par contre, l'exportation y est plus rare (2,3\% contre $11,6 \%)$. Enfin, les situations de forts déséquilibres sont à peu près aussi fréquentes pour les services (3\%) que pour l'industrie (4\%).

\section{Conclusion}

Plusieurs évaluations peuvent être faites sur cette recherche. Sans prétendre fournir un instrument parfait pour l'analyse, les profils types ont le mérite de proposer une classification qui complète l'approche sectorielle sans s'opposer ni se substituer à elle. Le regard porté sur les secteurs par ce moyen differre fondamentalement de l'approche sectorielle classique. En étudiant les entreprises, 
unités décisionnelles élémentaires composant un secteur, les profils permettent une «radiographie» beaucoup plus précise et nuancée que ce que permettent la plupart des approches qui agrègent les données de chaque secteur.

Il faut s'interroger sur ce à quoi correspondent les profils types. Les archétypes repérés correspondent bien à ce que l'on pourrait appeler des situations stratégiques, même si l'on ne peut prétendre qu'ils aient couvert le champ des possibles, ni exhumé toutes les catégories pertinentes pour le domaine des services. Nous sommes partis de critères dont la signification stratégique était plus évidente pour l'industrie. Fallait-il de nouveaux critères pour le champ des services? Le contexte des services, a priori différent de l'industrie, plaidait en ce sens : la relation des services à leur marché est différente, leur croissance passe, sans doute, par une multilocalisation plus que par des «ventes » à distance. Pour une firne de services, le développement nécessite des choix en termes de constitution de réseaux, de standardisation du concept, de sophistication de l'offre, de construction du concept autour de la compétence, du savoirfaire ou, différemment, de la technicité, de l'équipement matériel de la firme. Mais établir de nouveaux indicateurs n'est pas aussi évident que cela peut paraître a priori: la moitié ou les deux tiers des firmes de services n'ont pas répondu aux questions de l'enquête qui auraient permis d'avancer dans ce domaine. Les données comptables sont par contre toujours exposées. En fin de compte, les résultats obtenus intègrent un grand nombre de caractères spécifiques aux stratégies de services. Les profils types nous montrent des façons différentes de produire et de développer un service. Pour les services, une interdépendance existe entre le service proposé (et, donc, le secteur et la branche dans lesquels on opère) et la façon dont la prestation est réalisée. Mais il est significatif de noter que, malgré tout, les profils types traversent tout le champ sectoriel et que l'on peut trouver des firmes adoptant le profil «moyen», le profil «composite» ou le profil «compétence» dans tous les secteurs.

Une dimension reste absente de cette analyse, c'est la dimension géographique qui est déterminante pour l'entreprise de services. Nous l'avons explorée dans d'autres travaux (Léo et Philippe, 1991a), en comparant notamment les firmes qui ont opté pour un réseau d'établissements et les autres. Le rapprochement de ce travail avec l'analyse des profils permet d'émettre les observations suivantes:

- tant que le réseau d'établissements ne s'étend que sur une région (ou jusqu'à un département limitrophe), la répartition des profils stratégiques types reste très voisine de la moyenne, laissant une large place aux profils de type «moyen». On peut considérer que, pour ces dernières firmes, le réseau a constitué un axe de développement qui a exclu les autres. Par contre, dès que l'on considère les firmes dont les réseaux dépassent nettement l'échelle régionale, les profils «moyens» 
deviennent non spécifiques, alors que les profils «compétence», «composites » et «export» sont beaucoup plus fréquents. Cela est vrai pour les firmes parisiennes comme pour les firmes de province. Pour ces firmes, qu'elles soient PME ou non, il paraît bien qu'il existe une stratégie multi-axiale (savoir-faire, internationalisation et/ou réseau national d'établissements). Ce phénomène est caractéristique des services et rare chez les PME industrielles. 


\section{Annexe}

\section{Les ratios et critères utilisables}

Plus de quatre-vingts ratios ont été calculés pour chacune des 3970 firmes étudiées. La liste ci-dessous indique, sans préciser les variantes, quels sont les principaux ratios disponibles pour la plupart des firmes. Ils ont été utilisés pour typer ou caractériser les profils types:

1) Utilisation et gestion de la main-d'œuvre:

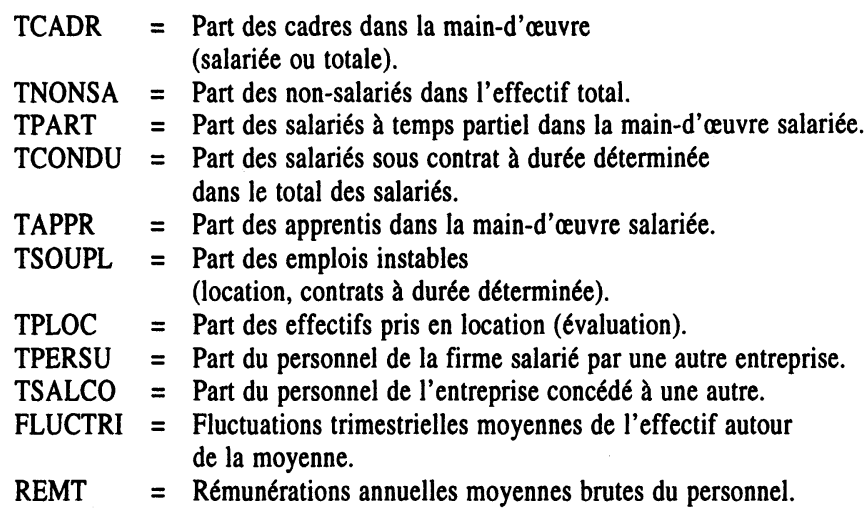

2) Importance et développement du capital:

$\begin{array}{ll}\text { IKE } & =\text { Immobilisations moyennes par effectif employé. } \\ \text { IKV } & =\text { Immobilisations moyennes par franc de valeur ajoutée. } \\ \text { AGEK } & =\text { Immobilisations moyennes par franc d'amortissement. } \\ \text { DYNTEC } & =\text { Indicateur de dynamisme technologique. } \\ \text { TINVV } & =\text { Part de la valeur ajoutée consacrée à l'investissement. } \\ \text { TINVK } & =\text { Part de l'investissement dans le capital. } \\ \text { INVFON } & =\text { Part des investissements consacrés aux facteurs fonciers. } \\ \text { INVOUT } & =\text { Part des investissements consacrés aux machines } \\ & \text { et à l'outillage. } \\ \text { INVTRA } & =\text { Part des investissements consacrés aux moyens de transport. } \\ \text { TOUTN } & =\text { Part des investissements-machines qui sont neufs. }\end{array}$

3) Combinaison productive, performances et résultats :
VALOR = Part du chiffre d'affaires qui est la valeur ajoutée par la firme.
PRODUCT $=$ Valeur ajoutée par effectif employé (moyenne).
TEXP = Part du chiffre d'affaires réalisé à l'étranger.
TXSST = Part du chiffre d'affaires rétrocédée à des confrères.
TSTM = Poids des stocks moyens par rapport au chiffre d'affaires.
RES/K = Taux de rendement (brut ou net) du capital immobilisé.
RES/V = Taux de résultat (brut ou net) d'exploitation rapporté à la valeur ajoutée. 


\section{Bibliographie}

BARCET, A., J. BONAMY et A. MAYÈrE (1983), L'économie des services aux entreprises, Lyon, Économie et humanisme.

BITNER, M.J. (1990), «Evaluating service encounters: the effects of physical surroundings and employee responses », Journal of Marketing, vol. 54, $\mathrm{n}^{\circ} 2$, p. $69-82$.

Czepiel, A.J., R.M. Solomon et F.C. SuRprenant (dir.) (1985), The Service Encounter. Managing Employee/Customer Interaction in Service Businesses, Lexington, Mass., Lexington Books.

Coffey, J.W. et M. Polese (1984). « La localisation des activités de bureau et des services aux entreprises », Revue d'Économie Régionale et Urbaine, $\mathrm{n}^{\circ} 5$, p. 717-729.

EIGlier, P. et E. LANGEARD (1988), Servuction, le marketing des services, Paris, McGraw-Hill.

GRÖNROSS, C. (1988). «Service quality: the six criteria of good perceived service quality », Review of Business, vol. 9, hiver, p. 1-9.

HESKETT, L.J. (1990), «Rethinking strategy for service management », dans B.E. David, C.B. Richard et T.G. Cummings (dir.), Service Management Effectiveness, $2^{\mathrm{e}}$ éd., San Francisco, Jossey-Bass Publishers, p. 17-40.

HUPPERT, R. (1981), «Stratégies de développement des PMI françaises », Revue d'Économie Industrielle, $\mathrm{n}^{\circ} 17$, p. 26-41.

LENTZ, T.R. (1980), «Strategic capability : a concept and framework for analysis», Academy of Management Review, vol. 5, $\mathrm{n}^{\circ}$ 2, p. 225-234.

LÉo, P.Y. (1986), «Contribution à un système d'information sur les PMI régionales à partir d'une exploitation de l'EAE en termes de profils-types d'entreprises. Rapport pour le Commissariat Général au Plan », Aix-en-Provence, C.E.R.

LÉo, P.Y. (1987), «Lès milieux régionaux de PMI: une approche statistique et régionalisée des choix stratégiques des PMI à partir de l'EAE», Revue d'Économie Régionale et Urbaine, $\mathrm{n}^{\circ} 3$, p. 423-437.

LÉO, P.Y. et J. PHILIPPE (1991a), «Services aux entreprises et organisations multilocales ", Revue d'Économie Industrielle, ${ }^{\circ}$ 57, $3^{\mathrm{e}}$ trim., p. 46-63.

LÉO, P.Y. et J. PHILIPPE (1991b), «Services aux entreprises : profils économiques et organisation spatiale. Rapport pour le ministère de la Recherche et de la Technologie», Aix-en-Provence, C.E.R.

MilligAN, G.W. et M.C. COOPER (1983), «An examination of procedures for determining the number of clusters in a data set », College of Administrative Science Working Paper Series, $\mathrm{n}^{\circ}$ 83-51, Columbus, Ohio State University. 
PARASURAMAN, A. et al. (1988), "SERVQUAL: a multiple item scale for measuring consumer perceptions of service quality», Journal of Retailing, vol. 64, printemps, p. 12-40.

PHILIPPE, J. (1990), «Information et milieu économique : des ressources à mobiliser», dans P.Y. Léo, M.C. Monnoyer et J. Philippe (dir.) , PME stratégies internationales, Paris, Economica.

QUINN, B.J. (1988), «Technology in services : past myths and future challenges », Technological Forecasting and Social Changes, ${ }^{\circ} 34$, p. 327-350.

SCHNEIDER, B. (1973), «The perception of organisational climate: the customer view », Journal of Applied Psychology, vol. 57, n 3, p. 248-256.

SHOSTACK, G.L. (1987), «Service positioning through structural change», Journal of Marketing, vol. 51, janvier, p. 34-43.

ThOMAS, R.E.D. (1978), « Strategy is different in service businesses », Harvard Business Review, juillet-août, p. 158-165.

ZeIthamL, V.A. et al. (1990), Delivering Quality Service: Balancing Customer Perceptions and Expectations, New York, The Free Press. 